\title{
Editorial: Signal Processing for Beyond 5G Non-Terrestrial Networks
}

\author{
Carlos Mosquera ${ }^{1 *}$ and Pantelis-Daniel Arapoglou ${ }^{2}$ \\ ${ }^{1}$ atlanTTic Research Center, University of Vigo, Vigo, Spain, ${ }^{2}$ European Space Research and Technology Centre (ESTEC), \\ Noordwijk, Netherlands
}

Keywords: 5G, 6G, signal processing, non-terrestrial networks (NTNs), satellite communication (SATCOM)

\section{Editorial on the Research Topic}

\section{Signal Processing for Beyond 5G Non-Terrestrial Networks}

Signal processing has long been playing an essential role in wireless terrestrial and satellite communications. Terrestrial networks are deploying new $5 \mathrm{G}$ based networks in various frequency bands, some of those in millimetre waves, the traditional realm of satellite networks, so that flexible mechanisms of sharing and coexistence will become more relevant. At the same time, the advent of new non-terrestrial wireless solutions such as High Altitude Platforms or highly dense Low Earth Orbit (LEO) constellations is positioning nonterrestrial networks (NTN) for a more decisive role in global data provision and ubiquitous coverage. However, a full integration between terrestrial and non-terrestrial networks is still not present; the 3rd Generation Partnership Project (3GPP) has concluded some initial studies on the role of NTN in 5G, a Work Item is expected to finalize in 2021, and NTN standardization in $5 \mathrm{G}$ is expected to take place in 3GPP Releases 17 and 18. It is likely that for some years to come, both networks will still remain complementary, with a potential real integration given by the timeframe of $6 \mathrm{G}$, where NTN is considered key in providing connectivity to rural and underprivileged areas.

There is a strong need for signal processing R\&D in the new NTN, which at the same time helps to close the bridge with terrestrial networks: the farther away we move from the physical layer, the more commonalities can be found between space and terrestrial segments. The sharing of radio frequency bands and the dynamic usage of resources allow to operationally reconfigure network connections. In this context, the signal processing in the transmitters, receivers, and network control systems face significant requirements to access frequency resources dynamically, detect interference, optimize the network configuration, and configure the radio resources operationally. Signal processing must be used to leverage the flexibility of resource allocation in NTN, including GEO, MEO and LEO satellites, aerial platforms and even unmanned aerial vehicles (UAV).

Four contributions have been included in this collection to illustrate the key role of signal processing at the interplay with networking, scheduling and antenna technology.

The paper "Precoding with Received-Interference Power Control for Multibeam Satellite Communication Systems" (Lagunas et al.) builds on classical zero-forcing based precoders to propose a new scheme to reduce the sensitivity to the user scheduling, making it also possible to operate under overloaded conditions. As the number of beams increases, and aggressive frequency reuse is desired to get the most out of the scarce available spectrum, techniques like that exposed in this paper can help to bridge the gap between user scheduling and physical layer methods.

The paper "Field Trial on 5G New Radio over Satellite" (Völk et al.) contributes with a practical testbed, featuring the use of a GEO satellite for backhauling a 5G mobile node. A commercial off-the-shelf 5G user terminal was connected to the core network through a mobile edge node operating on a satellite link on the Ku-band. Even though the lack of integrated standardization between terrestrial and satellite systems limits the performance, we can expect a tighter integration in beyond $5 \mathrm{G}$ and, most likely, $6 \mathrm{G}$ systems, so that even seamless operation making use of both terrestrial and satellite links will be possible. 
The paper "A Low-Complexity Reconfigurable Multi-Antenna Technique for non-Terrestrial Networks" (Maliatsos et al.) performs a joint transmit/receive beamforming with beam selection and combining for improving the performance of aerial communication systems. The implementation of this novel beamforming and pattern adaptation scheme is enabled by the use of Electronically Steerable Parasitic Array Radiators (ESPAR), properly designed for Ground Station to UAV links.

The paper "A Hybrid Beamforming Design for Massive MIMO LEO Satellite Communications" (Palacios et al.) presents a hybrid beamforming strategy based on an analog codebook; similarly to its use in terrestrial base stations, a DFT-type codebook is proposed to be used from LEO satellites, and room is left for the design of digital precoders able to mitigate the ensuing co-channel interference.

We can expect some frenzied activity during this decade to finally adopt the satellites as an essential element of the terrestrial communication networks, a long standing aspiration whose time may has come thanks to the right combination of commercial and technological factors.

\section{AUTHOR CONTRIBUTIONS}

All authors listed have made a substantial, direct, and intellectual contribution to the work and approved it for publication.

Conflict of Interest: The authors declare that the research was conducted in the absence of any commercial or financial relationships that could be construed as a potential conflict of interest.

Publisher's Note: All claims expressed in this article are solely those of the authors and do not necessarily represent those of their affiliated organizations, or those of the publisher, the editors and the reviewers. Any product that may be evaluated in this article, or claim that may be made by its manufacturer, is not guaranteed or endorsed by the publisher.

Copyright (c) 2021 Mosquera and Arapoglou. This is an open-access article distributed under the terms of the Creative Commons Attribution License (CC $B Y)$. The use, distribution or reproduction in other forums is permitted, provided the original author(s) and the copyright owner(s) are credited and that the original publication in this journal is cited, in accordance with accepted academic practice. No use, distribution or reproduction is permitted which does not comply with these terms. 